\title{
Isolated Tricuspid Valve Surgery - Repair Versus Replacement: a Meta-analysis
}

Panagiotis Sarris-Michopoulos ${ }^{1}$, Alejandro Macias ${ }^{2}$, Constantine Sarris-Michopoulos ${ }^{3}$, Palina Woodhouse ${ }^{4}$, Daniel Buitrago ${ }^{5}$, Tomas Salerno ${ }^{6}$, and Michael Magarakis ${ }^{7}$

${ }^{1}$ University of Miami Miller School of Medicine

${ }^{2}$ University of Miami Health System

${ }^{3}$ University of Georgia

${ }^{4}$ Vanderbilt University School of Medicine

${ }^{5}$ University of Miami Miller School of Medicine; Jackson Memorial Hospital; Department of Surgery, Division of Cardiothoracic Surgery

${ }^{6}$ university of miami miller school of medicine and jackson memorial hospital

${ }^{7}$ Miami VA Medical Center - University of Miami Miller School of Medicine

August 10, 2021

\begin{abstract}
Objective: There is paucity of data on outcomes after isolated tricuspid valve surgery. This meta-analysis aims to compile available data on isolated tricuspid valve surgery and compare isolated tricuspid valve repair (iTVr) with isolated tricuspid valve replacement (iTVR) to elucidate outcomes after tricuspid valve surgery. Methods: A literature search of 6 databases was performed. The primary outcomes was 30-day mortality. Secondary outcomes were early stroke, post-op pacemaker placement, and tricuspid reoperation within 5 years. Publication bias was explored using the funnel plot. Results: Ten retrospective studies involving 1407 patients (iTVr group $=779$ patients and iTVR group $=628$ patients) were included. A cumulative analysis demonstrated a significant difference favoring iTVr for 30-day mortality [odds ratio - 10 studies (95\% confidence interval) 0.34 (0.18-0.66)]; $4.7 \%$ versus $12.6 \%$, for iTVr and iTVR, respectively. Post-op pacemaker placement favored iTVr [odds ratio -6 studies (95\% confidence interval) 0.37 (0.18-0.77)]. Although stroke rates and TV reoperation favored iTVr, they did not reach statistical significance. No publication bias was identified. Conclusions: This meta-analysis demonstrates that iTVr has better 30-day mortality and fewer permanent pacemaker placements. Etiology and severity of TR, as well as careful patient selection remain the most important factors for optimal outcomes.
\end{abstract}

\section{Hosted file}

iTVr_Vs_iTVR 7.19.2021.docx available at https://authorea.com/users/388689/articles/533525isolated-tricuspid-valve-surgery-repair-versus-replacement-a-meta-analysis 